\title{
DIGITAL SOLUTION FOR FAST EVALUATION OF THE POWER FACTOR IN POWER NETWORKS
}

\author{
Adrian GLIGOR ${ }^{1}$, Raul MUNTEAN ${ }^{2}$, Cristian-Dragoș DUMITRU ${ }^{3}$ \\ ${ }^{1,2,3}$ Petru Maior University of Tirgu-Mures \\ Nicolae Iorga st., no. 1, Tirgu-Mures, 540088, Romania \\ 1adrian.gligor@ing.upm.ro \\ 2muntean.raul@stud.upm.ro \\ 3 cristian.dumitru@ing.upm.ro
}

\begin{abstract}
Power quality evaluation involves measurements and determinations of some power quality indicators. One of these indicators is represented by the power factor. The importance of the power factor as an indicator of power quality, both theoretical and practical, implies its determination or direct measurement as a necessity, especially in industrial environments where the optimization of energy costs is a primary target. Considering these, the determination of this power quality indicator implies multiple or sophisticated measurement tools. As a result, the present paper aims to present a solution for an easier evaluation of the power factor. The proposed solution can be integrated in numerical control systems for reactive power monitoring or in supervision and control systems in order to extend the applicability for the automatic power factor improvement. The proposed solution is characterized by simplicity and a low cost of implementation, representing an effective learning tool in the professional training in the electricity field. The configuration of the measurement circuit was designed to allow the measurement of a wide range of the power factor, starting from the case of inductive consumers to the case of capacitive consumers, noting that in the case of strongly deformed regimes the results shows significant deviations from the expected ones fact that is extensively exposed and motivated in the paper.
\end{abstract}

Key words: power networks, power quality, power factor, harmonic pollution, data acquisition.

\section{Introduction}

One of the main goals in nowadays industry is represented by the production costs optimization. In many sectors a significant part of these costs is attributed to electricity consumption. On the other hand, for reasons regarding the efficient use of energy resources and environmental protection, a series of regulations regarding the power supply and the use of electricity were introduced. The two mentioned directions led to a common objective for the power supplier and the power consumer regarding the consumption of electricity monitoring and optimization [1]. However, to ensure an equal approach a set of power quality indicators had to be implemented.

One of these power quality indicators is represented by the power factor expressing reactive power flow relative to the apparent power consumed [2], [3].
By knowing this indicator the associated losses recorded costs in power networks correlated with the active power transportation can be evaluated. This indicator can be used in the monitoring of the energetic behavior of power networks and consumers to identify and provide the means for achieving a lower cost for the supplied electricity. In this way, the industrial consumers will benefit of a useful measure in assessing the effectiveness and safety in the use of electricity.

Starting from this practical issue, the present paper studied and designed a numerical solution for the power factor determination.

In order to evaluate performances and viability of the solution a prototype was implemented and tested in the laboratory.

The present paper illustrates the theoretical formalism and the design of the proposed solution for measuring the power factor. Based on experimental 
results some conclusions and observations were made.

\section{Power Factor in Power Networks}

The power factor has many definitions, among them the IEEE standard 1459-2010 being widely accepted [7],[8]. The power factor is an important parameter in assessing the efficiency of power transmission. It is expressed as the ratio of active power $P$ also called real power and apparent consumed power $S$ of the circuit:

$$
P F=\frac{P}{S}
$$

The relation (1) shows that the power factor indicates the proportion of active power from apparent power.

The above definition is not generally suitable for all situations, so the power factor is treated depending on the type of the permanent regime that is evaluated [9].

Thus, in a permanent sinusoidal regime:

$$
P F=\frac{P}{S}=\frac{S \cdot \cos \varphi}{S}=\cos \varphi
$$

where $\varphi$ represents the phase angle between the voltage and current phasors. The possible values are between 0 and 1, inductive or capacitive, as voltage phasor is before or after the current phasor.

In non-sinusoidal regime the power factor reflects the displacement components and also the distortion [4]. In order to differentiate the two situations the distortion and then total power factor were introduced [7].

The distortion power factor can be computed from the equation [5]:

$$
D P F=\frac{1}{\sqrt{1+T H D_{I}^{2}}}
$$

where $T H D_{I}$ denote the total harmonic distortion of the load current.

Accordingly the total power factor is given by the following equation:

$$
P F_{T O T}=\cos \varphi \cdot \frac{1}{\sqrt{1+T H D_{I}^{2}}}=P F \cdot D P F
$$

This equation shows that the total power factor is directly proportional to the power factor in permanent sinusoidal regime and the above defined distortion power factor.

\section{Principle of Measurement}

In permanent sinusoidal regime waveforms are represented in amplitude and time. In this case power factor represents the time difference between current and voltage waveforms. The proposed device measures the time at which the current and voltage passes through the zero point and compares the time values.

$$
\cos \varphi=\cos 2 \pi f \Delta t
$$

where $\Delta t$ represents the time difference between the zero cross of voltage and current signals; $f$ is the mains frequency.

\section{Digital Device for Power Factor Evaluation}

Using the measurement principle presented in the previous paragraph, a simple block diagram is proposed to be designed as shown in Figure 1.

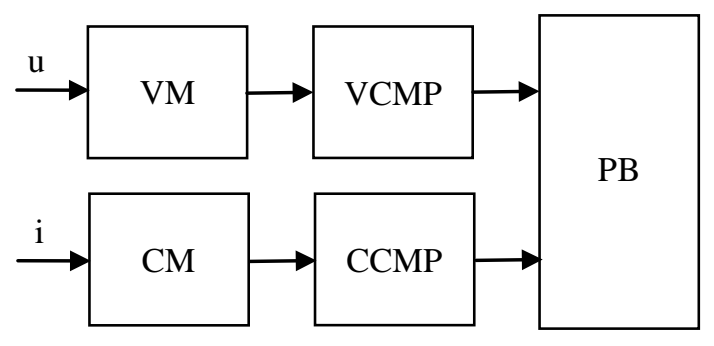

Fig. 1: Power factor measurement bloc diagram

The proposed solution for power factor determination requires a voltage $u$ and current $i$ measurement in the analyzed circuit by using voltage $\mathrm{VM}$ and current CM sensors. Instantaneous values of the two measured parameters are applied to the voltage VCMP and current CCMP comparator blocks to determine the zero crossing of the two parameters. The comparators outputs are applied to the processor unit $\mathrm{PB}$ where the mains frequency and power factor are determined based on relation (2).

\section{Implementation of the Proposed Solution}

Based on the described architecture the electronic schematic proposed is shown in Figure 2.

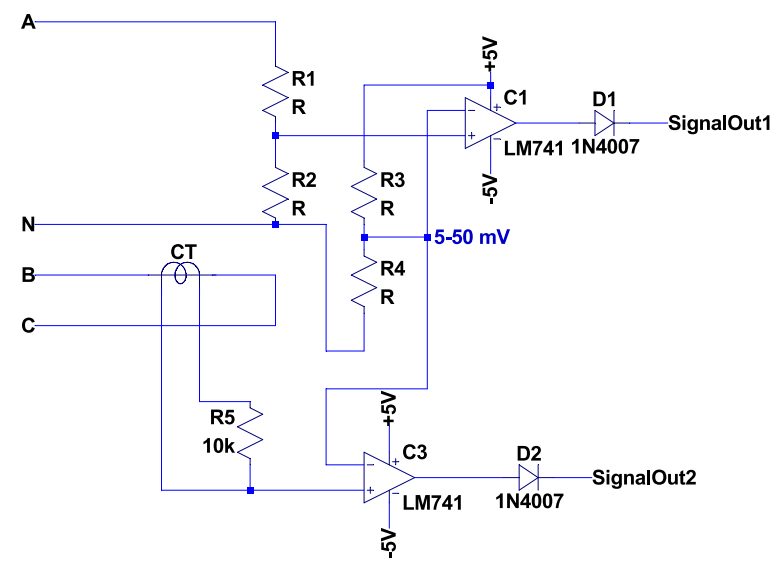

Fig. 2: Electronic circuit diagram

In the electronic diagram from Figure 2, A and $\mathrm{N}$ are voltage inputs and $\mathrm{B}$ and $\mathrm{C}$ are current inputs. The resistors $R_{1}$ and $R_{2}$ are dimensioned to the DC voltage test measured, but in practice, are replaced with a voltage transformer. If the device is used as a teaching module the current measurement circuit CT can be replaced with a properly sized shunt. For low voltage schemes a LEM55 type device can be used 
successfully. For high value currents the device must be replaced with an appropriate current transformer measure.

The SignalOut1 and SignalOut2 exits represent the inputs of the calculation block. The waveform of these signals can be observed in Figure 3.

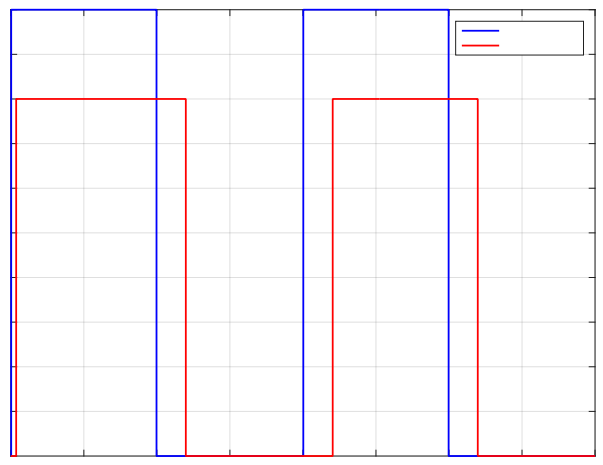

Fig. 3: Waveforms of the SignalOut1 and SignalOut2 signals

In Fig 3 the amplitude of the signals is represented at different levels in order to differentiate them.

\section{Software Computation of the Power Factor}

In this case the computation hardware platform consists from an ATMEGA328p microcontroller type. The calculation of the power factor is performed by an application written in $\mathrm{C} / \mathrm{C}++$. The computation algorithm consists in determining the time difference between zero crossings of voltage and current signals.

In order to get accurate results the zero crossing detection is realized by interrupt requests.

The algorithm sequence steps are:

Data: calculation period Tc

Result: PF, mains frequency

Hardware initializations

while true

repeat

zero cross detection for voltage

until no. of zero-cross detection is 2

Compute mains frequency (period)

do

Detect zero-crossing for voltage

Detect zero-crossing for current

if time difference $>\mathrm{T} / 2$ then

compute capacitive $\mathrm{PF}$

else

compute inductive PF

end

while Tc expires

\section{Power Factor Measurement Evaluation}

In order to test the proposed solution a series of measurements with the proposed device and with a Qualistar type power analyzer (CA 8335) produced by Chauvin Arnoux were performed.

Measurements consisted in power factor determination for different types of consumers, such as a resistive consumer, an inductive consumer, and a capacitive consumer. A summary of results is presented in Table 1 . The measurements performed with the proposed device are presented in "Measure 1 " column, while those obtained with the power analyzer are presented in "Measure 2" column.

Table 1: Experimental measurements

\begin{tabular}{|l|c|c|c|}
\hline $\begin{array}{l}\text { Load } \\
\text { Type }\end{array}$ & Measure 1 & Measure 2 & $\begin{array}{c}\text { Deviation } \\
{[\%]}\end{array}$ \\
\hline Resistive & 0 & 0 & 0 \\
\hline Inductive & 0.77 & 0.788 & 2.28 \\
\hline Capacitive & 0.979 & 0.9659 & 1.35 \\
\hline
\end{tabular}

The obtained results show a small difference between the two methods of measurement.

In order to determine the cause of deviations in the power factor measurement process the voltage and current wave forms were recorded both with an oscilloscope and with the Qualistar device. The Figures 4, 5 and 6 show the results recorded with the oscilloscope while Figures 7, 8 and 9 originates from the power quality analyzer.

Between the two sets of measurements we observe some differences that are mainly due to the perturbations and the non-linear circuit elements such as the supply transformer, induction motor, etc.

For a better illustration of the obtained results in Figures 10, 11 and 12 are presented the phasor diagram for three studied distinct case of a resistive, inductive and capacitive load.

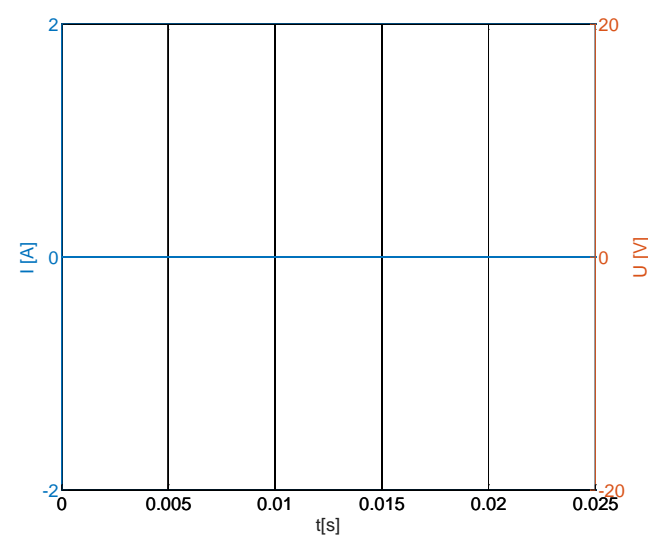

Fig. 4: Voltage and current waveforms in case of resistive load

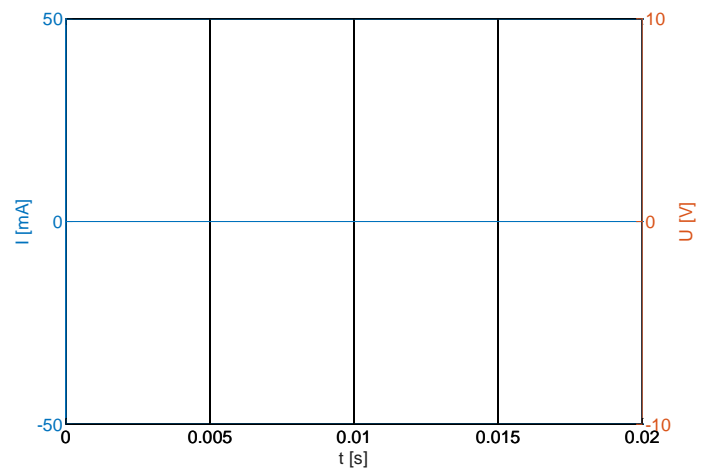

Fig. 5: Voltage and current waveforms in case of inductive load 


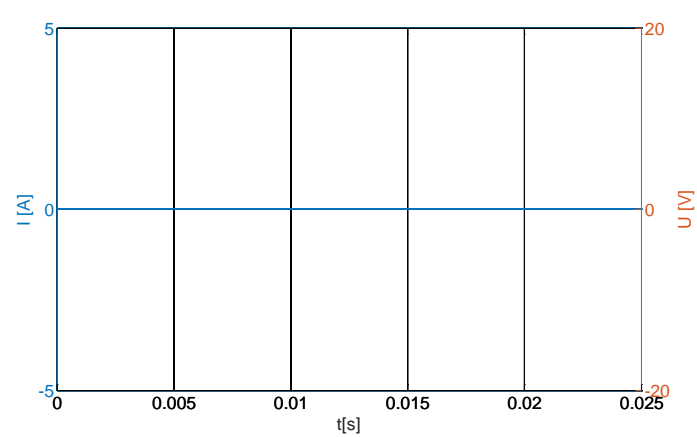

Fig. 6: Voltage and current waveforms in case of capacitive load

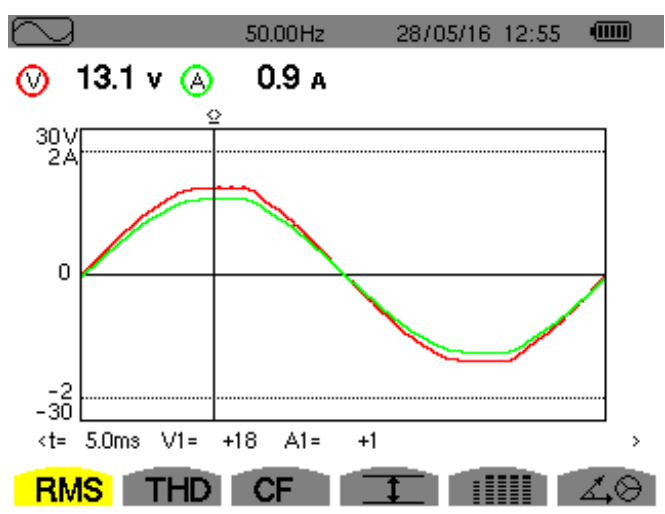

Fig. 7: Voltage and current waveforms in case of resistive load by power quality analyzer device

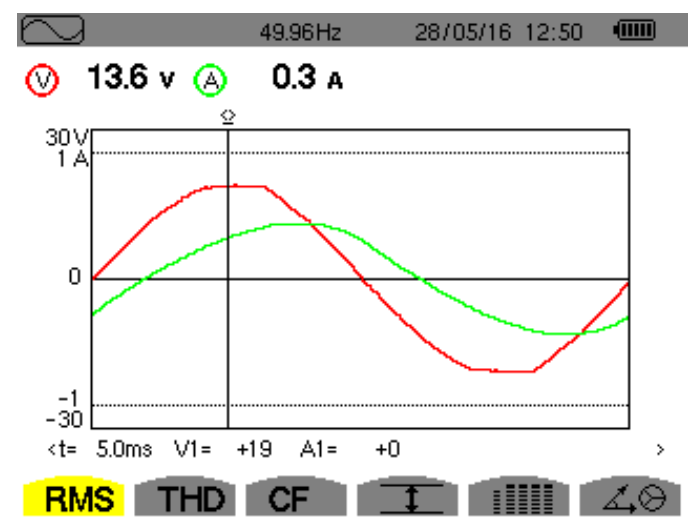

Fig. 8: Voltage and current waveforms in case of inductive load by power quality analyzer device

The proposed device provides good results but care must be taken in case of non-linear loads. As we can see from equation (4) power factor calculation is less precise because power factor is not evaluated considering the voltage and current waveform distortion.

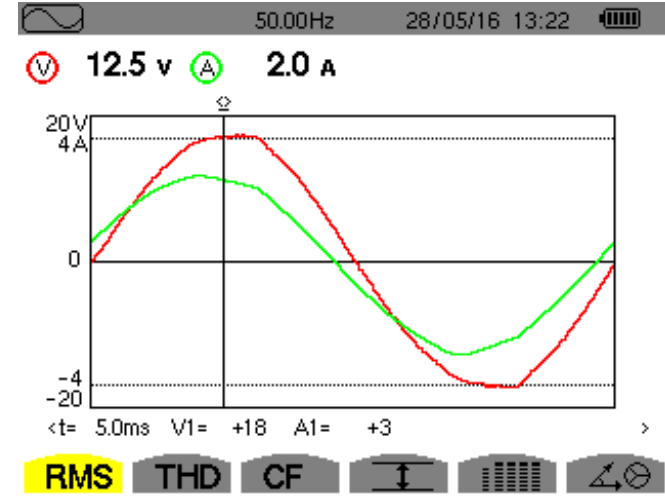

Fig. 9: Voltage and current waveforms in case of capacitive load by power quality analyzer device

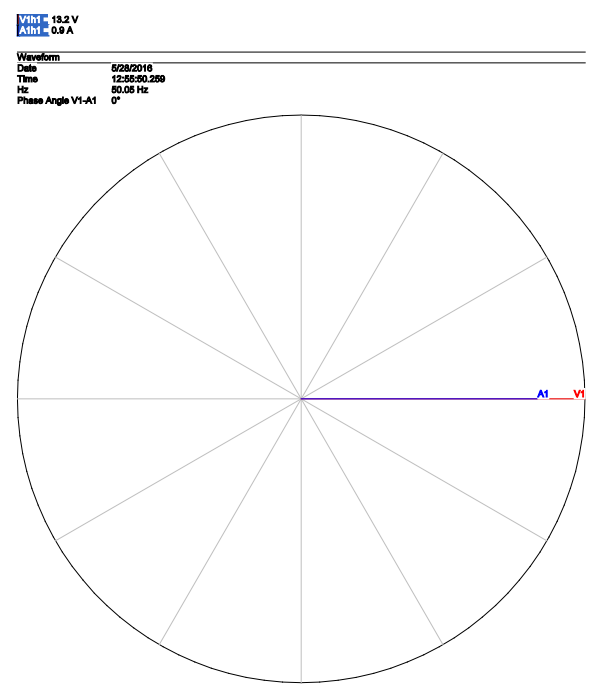

Fig. 10: The phasor diagram in case of resistive load supplied by power quality analyzer device

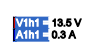

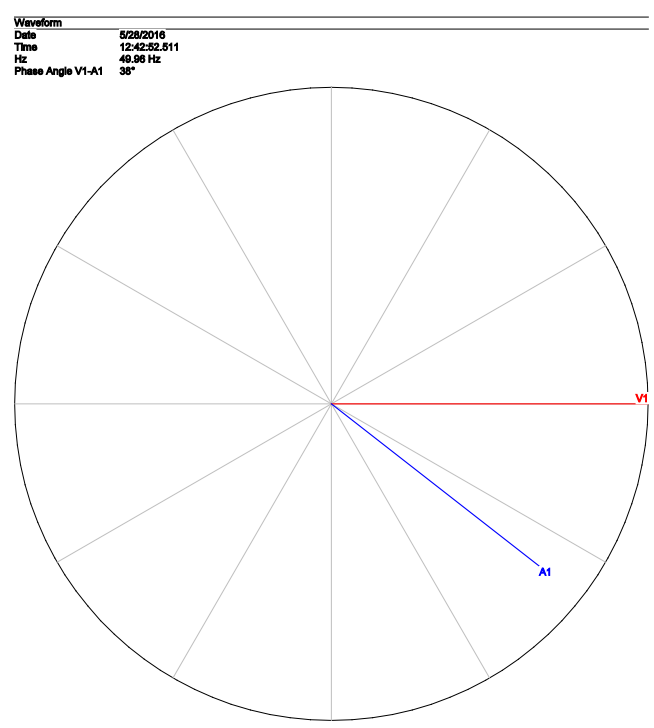

Fig. 11: The phasor diagram in case of inductive load supplied by power quality analyzer device 


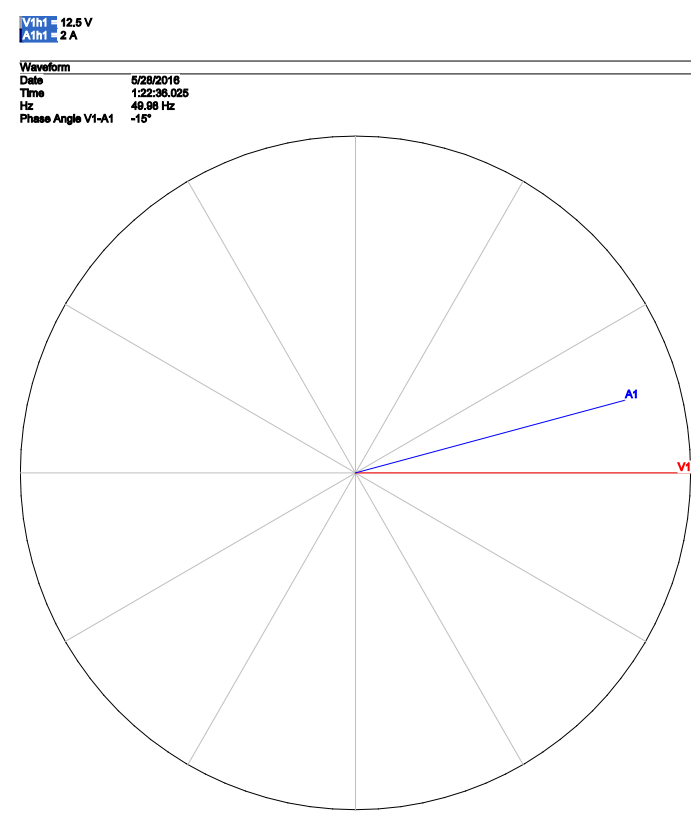

Fig. 12: The phasor diagram in case of capacitive load supplied by power quality analyzer device

\section{Conclusions}

Nowadays, the reducing of energy consumption as a result of very strict environmental and power quality standards is possible by a careful monitoring of the consumers load profile. Due to the high costs the use of consumers who do not integrate strictly within the fixed standards indicators is currently adopted. For this reason these consumers can generate additional costs inside the power network or in the industrial or distribution and transmission network on which they are connected. In these cases a monitoring solution is imperative. Appliances often dedicated to this purpose can be expensive or too complex compared to the followed objective. Thus the proposed device can represent a viable solution into a centralized system for monitoring the quality factor on an industrial platform.

It should be mentioned that the presented solution has a number of limitations caused by the chosen calculation algorithm and its replacement will not provide an appropriate level related to the required computing power.

For determinations in a range of error of 5\%, the solution is acceptable. For a better precision more efficient alternatives are recommended. As an immediate solution the replacement based on a DSP processing unit and of the corresponding algorithms is recommended.

The performed study shows that in the case of the linear load the determination of the power factor is an easy task, but for nonlinear consumers the situation changes. For moderate nonlinearities the accepted margin of error operation of the device is acceptable, but, if the sources are strongly nonlinear such as power switchers of the power converters, the results are not consistent on the one hand due to the multiple zero-crossing of the current signal across the period chosen for the detection and on the other hand due to significant component of DPF.

\section{Acknowledgement}

This paper is based upon work supported by the Energy and Electrotechnologies Management research centre.

\section{References}

[1] Gligor, A. (2014), Asigurarea calităţii energiei electrice în instalațiile electrice poluate armonic (Power Quality Assurance in Harmonic Polluted Electrical Installations), "Petru Maior" University Press, 2014, pp. 10-23.

[2] Lee, K. (2008), Power Quality Analysis and New Harmonic and Unbalance Control of Modern Adjustable Speed Drives Or Uninterruptible Power Systems Under Nonideal Operating Conditions, University of Wisconsin-Madison, pp. 19-24.

[3] Mikkili, S., Pandapp A.K. (2015). Power Quality Issues: Current Harmonics, CRC Press, 31-36.

[4] Das, J. C. (2015). Power System Harmonics in Power System Harmonics and Passive Filter Designs, John Wiley \& Sons, pp. 1-29.

[5] Dimitrijević, M.A., Litovski, V.B.(2006), Power Factor and Distortion Measuring for Small Loads Using USB Acquisition Module, Conference REV2006, 29/30 June 2006 Maribor, Slovenia, pp. 1-5.

[6] Baggini, A. and Hanzelka, Z. (2008), Voltage and Current Harmonics, in Handbok of Power Quality, Baggini, A. (ed), Jhon Wiley and Sons, pp. 214-216

[7] Fuchs, E. and Masoum, M.A.S. (2015), Interaction of Harmonics with Capacitors in Power Quality in Power Systems and Electrical Machines, Academic Press, pp. 434-436

[8] Bollen M.H., Gu I. (2006), Power Quality Indices. Power Quality Indices in Signal Processing of Power Quality Disturbances, John Wiley \& Sons, pp. 217-220.

[9] Mansour, A., Chengning, Z., and Nasry, H. (2013). Measurement of power components in balanced and unbalanced three-phase systems under nonsinusoidal operating conditions by using IEEE standard 1459-2010 and Fourier analysis. In Techn. Adv. in Electrical, Electronics and Computer Engineering (TAEECE), IEEE Intl. Conf., pp. 166-171. 